\title{
4-PSK Balanced STTC with two transmit antennas
}

\author{
Thi Minh Hien Ngo, Gheorghe Zaharia, Stéphane Bougeard, Jean François Hélard \\ Institute for Electronics and Telecommunications of Rennes (IETR) - UMR CNRS 6164 \\ INSA - 20 avenue des Buttes de Coësmes, 35043 Rennes, France \\ Email: Minh-Hien.Ngothi@ens.insa-rennes.fr
}

\begin{abstract}
In this paper we introduce a new class of space-time trellis codes (STTC). We call them Balanced STTC (B-STTC) because the points of the constellation are used with the same probability. Comparing to known codes, the balanced codes offer the best performance. Therefore, the systematic search for good codes can be reduced to this class. We present here the design of the 4-PSK balanced STTC with 2 transmit antennas. A complete list of the best 4-state and several 16-state good balanced codes are also given.
\end{abstract}

\section{INTRODUCTION}

Barring complexity issues, STTC have been shown to outperform other space-time coded systems. Tarokh et al. [1] introduced the concept of STTC as an extension to the conventional time-convolutional coding and derived criteria to obtain optimal codes. For quasistatic flat Rayleigh or Rician channels, performance is shown to be determined by the diversity advantage quantified by the rank of certain matrices and by the coding advantage that is quantified by the determinants of these matrices. For rapidly changing flat Rayleigh channels, performance is determined by the diversity advantage quantified by the generalized Hamming distance of certain sequences and by the coding advantage that is quantified by the generalized product distance of these sequences which are constructed from pairs of distinct codewords. In [2] Chen introduced the trace criterion which governs the coding for systems with a great product of the numbers of transmit and receive antennas.

Based on above criteria, many different STTC for 2 transmit antennas have been found by a systematic code search [3]-[9].

Until now, no efficient construction method of STTC has been proposed. The main contribution of this paper is the description of a method of construction of a new class of Balanced STTC (B-STTC) which offers the best performance. Therefore, the systematic search for good codes can be reduced to this class.

The rest of the paper is organized as follows. Section II briefly describes the STTC. Their performance criteria, depending on the channel properties, are presented in Section III. The new class of balanced codes is introduced in Section IV and their properties are listed in Section V. The design of 4-PSK balanced space-time trellis codes for 2 transmit antennas is described in Section VI. Finally, it is shown in Section VII that the best B-STTC outperform or equal previously known codes.

\section{SPACE TIME TRELLIS CODING}

We consider the case of 4-PSK space-time trellis encoder as shown on Fig.1.
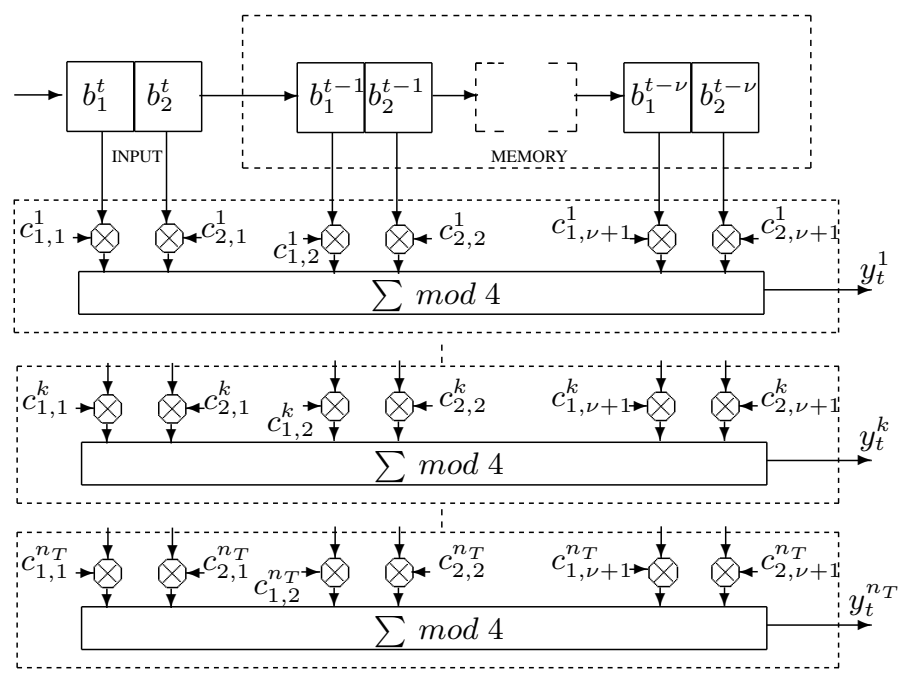

Fig. 1. Space-time trellis encoder with 4-PSK and $n_{T}$ transmit antennas

In the general case of a $2^{n}$-PSK modulation, this encoder is composed of one input block of $n$ bits and $\nu$ memory blocks of $n$ bits. At each time $t \in \mathbb{Z}$, all the bits of a block are replaced by the $n$ bits of the previous block. The $i^{t h}$ bit $b_{i}^{t-j+1}, i=1 \ldots n$, of the $j^{t h}$ block, $j=1 \ldots \nu+1$, is associated to $n_{T}$ multiplier coefficients $c_{i, j}^{k} \in \mathbb{Z}_{2^{n}}$, $k=1 \ldots n_{T}$ where $n_{T}$ is the number of transmit antennas. A ST trellis encoder is thus classically defined by its generator matrix $\mathbf{C}$ of $n_{T} \times n(\nu+1)$ coefficients:

$$
\mathbf{C}=\left[\begin{array}{ccccccc}
c_{1,1}^{1} & \ldots & c_{n, 1}^{1} & \ldots & c_{1, \nu+1}^{1} & \ldots & c_{n, \nu+1}^{1} \\
\vdots & & & \ldots & & & \vdots \\
c_{1,1}^{k} & \ldots & c_{n, 1}^{k} & \ldots & c_{1, \nu+1}^{k} & \ldots & c_{n, \nu+1}^{k} \\
\vdots & & & \ldots & & & \vdots \\
c_{1,1}^{n_{T}} & \ldots & c_{n, 1}^{n_{T}} & \ldots & c_{1, \nu+1}^{n_{T}} & \ldots & c_{n, \nu+1}^{n_{T}}
\end{array}\right]
$$

The encoder outputs for the $k^{t h}$ antenna are computed as

$$
y_{t}^{k}=\sum_{i=1}^{n} \sum_{j=1}^{\nu+1} b_{i}^{t-j+1} c_{i, j}^{k} \quad \bmod 2^{n}
$$


where $y_{t}^{k} \in \mathbb{Z}_{2^{n}}$ represents the index of the $2^{n}$-PSK symbol $s_{t}^{k}=e^{\jmath y_{t}^{k} \frac{\pi}{2}}$ sent to the $k^{t h}$ antenna. The modulated streams for all antennas are then transmitted simultaneously.

At the receiver, each one of the $n_{R}$ antennas collects the superpositions of the faded replicas of the $n_{T}$ transmitted symbols. Each link between one transmit antenna and one receive antenna is assumed to experience statistically independent flat Rayleigh fading. Therefore, we can use the following model of the received signal:

$$
r_{t}^{l}=\sum_{k=1}^{n_{T}} h_{k l, t} s_{t}^{k}+n_{t}^{l}
$$

where $r_{t}^{l}$ is the signal received at antenna $l$ at time $t$; $h_{k l, t}$ is the complex path gain from transmit antenna $k$ to receive antenna $l$ at time $t ; s_{t}^{k}$ is the transmitted complex symbol corresponding to $y_{t}^{k}$; and $n_{l}^{t}$ is the AWGN sample for receive antenna $l$ at time $t$. The noise samples are independent samples of a zero-mean complex Gaussian random variable with spectral density $N_{0} / 2$ per dimension. Maximum likelihood decoding is then used to extract the transmitted codeword.

\section{DESIGN CRITERIA}

Design criteria have been proposed in [1] [2] to exploit the $n_{T} n_{R}$ spatial diversity order and to offer optimal coding gain. Cases of slow and fast Rayleigh fading are mainly studied. The transmitted $n_{T}$ dimension symbols $\mathbf{s}_{t}=\left[s_{t}^{1} s_{t}^{2} \ldots s_{t}^{n_{T}}\right]^{\mathrm{T}}$, where $[\cdot]^{\mathrm{T}}$ denotes the transpose operator, are assumed to be grouped in a frame of length $L_{f}$.

A channel is said to be a slow Rayleigh fading channel if, during a transmission of a frame, the complex path gains $h_{k l}$ do not change with time $t$, but are independent from one frame to the next one. In the case of a fast Rayleigh fading channel, the complex path gains $h_{k l, t}$ are independent from a $n_{T}$ dimension symbol to the next one. For each case, criteria are derived from the minimization of the Pairwise Error Probability (PEP), i.e. the probability of transmitting the $n_{T} \times L_{f}$ dimension coded frame $\mathbf{S}=\left[\mathbf{s}_{t} \mathbf{s}_{t+1} \ldots \mathbf{s}_{t+L_{f}-1}\right]$ and deciding erroneously in favour of another $n_{T} \times L_{f}$ dimension coded frame $\mathbf{E}=\left[\mathbf{e}_{t} \mathbf{e}_{t+1} \ldots \mathbf{e}_{t+L_{f}-1}\right]$. The $n_{T} \times n_{T}$ product matrix $\mathbf{A}=\mathbf{B B}^{*}$ is introduced where $\mathbf{B}^{*}$ denotes the hermitian of the $n_{T} \times L_{f}$ difference matrix $\mathbf{B}=\mathbf{E}-\mathbf{S}$ described as follows :

$$
\mathbf{B}=\left[\begin{array}{ccccc}
e_{t}^{1}-s_{t}^{1} & \ldots & e_{t+q}^{1}-s_{t+q}^{1} \ldots & e_{t+L_{f}-1}^{1}-s_{t+L_{f}-1}^{1} \\
\vdots & \vdots & \ldots & \vdots & \vdots \\
e_{t}^{n_{T}}-s_{t}^{n_{T}} & \ldots & e_{t+q}^{n_{T}}-s_{t+q}^{n_{T}} \ldots & e_{t+L_{f}-1}^{n_{T}}-s_{t+L_{f}-1}^{n_{T}}
\end{array}\right]
$$

\section{A. The case of slow Rayleigh fading}

In the case of slow Rayleigh fading channels, two criteria have been proposed in [1]. Firstly, in order to maximize the diversity advantage, the product matrix $\mathbf{A}$ has to be full rank over all possible pairs $(\mathbf{E}, \mathbf{S})$. Since the maximal value of $\operatorname{rank}(\mathbf{A})$ is $n_{T}$, the achieved spatial diversity order is then equal to the product $n_{T} n_{R}$. Secondly, in order to maximize the coding gain, the minimum product $\prod_{k=1}^{\operatorname{rank}(\mathbf{A})} \lambda_{k}$, where $\lambda_{k}$ are the nonzero eigenvalues of $\mathbf{A}$, has to be maximized by choosing the generator matrix $\mathbf{C}$. If $\operatorname{rank}(\mathbf{A})=n_{T}$, we can note that

$$
\operatorname{det}(\mathbf{A})=\prod_{k=1}^{n_{T}} \lambda_{k}=\prod_{k=1}^{n_{T}}\left(\sum_{q=0}^{L_{f}-1}\left|e_{t+q}^{k}-s_{t+q}^{k}\right|^{2}\right)
$$

The codes that can achieve the best performance present the greatest value of the minimum product $\prod_{k=1}^{\operatorname{rank}(\mathbf{A})} \lambda_{k}$. For a full rank product matrix, the achieved coding advantage is then equal to $\operatorname{det}(\mathbf{A})^{\left(1 / n_{T}\right)}$.

\section{B. The case of fast Rayleigh fading}

In the case of fast Rayleigh fading channels, different criteria have been obtained in [1]. Tarokh defines the Hamming distance $d_{H}(\mathbf{E}, \mathbf{S})$ between two coded frames $\mathbf{E}$ and $\mathbf{S}$ as the number of time intervals for which $\left|\mathbf{e}_{t}-\mathbf{s}_{t}\right| \neq 0$. In order to maximize the diversity advantage over fast Rayleigh fading channels, the minimal Hamming distance $d_{H}(\mathbf{E}, \mathbf{S})$ computed over all pairs of coded frames has to be maximized by choosing the generator matrix $\mathbf{C}$. The achieved spatial diversity order is then equal to the product $d_{H}(\mathbf{E}, \mathbf{S}) \cdot n_{R}$. In the same way, Tarokh introduces the product distance $d_{p}^{2}(\mathbf{E}, \mathbf{S})$ as the product of euclidian distance between the $L_{f} n_{T}$-dimension symbols composing the coded frames $\mathbf{S}$ and E. The product distance is written

$$
d_{p}^{2}(\mathbf{E}, \mathbf{S})=\prod_{q=0}^{L_{f}-1}\left(\sum_{k=1}^{n_{T}}\left|e_{t+q}^{k}-s_{t+q}^{k}\right|^{2}\right)=\prod_{q=0}^{L_{f}-1} d_{\mathbf{E}}^{2}\left(e_{t+q}, s_{t+q}\right)
$$

In order to maximize the coding gain, the minimum product distance $d_{p}^{2}(\mathbf{E}, \mathbf{S})$ computed over all pairs $(\mathbf{E}, \mathbf{S})$ has to be maximized by choosing the generator matrix $\mathbf{C}$. The achieved coding advantage is then equal to $d_{p}^{2}(\mathbf{E}, \mathbf{S})^{1 / d_{H}(\mathbf{E}, \mathbf{S})}$.

\section{The case of a larger number of antennas}

In [2], Chen proposes a new criterion which is valid in the case of slow and fast Rayleigh fading channels since the product $\operatorname{rank}(\mathbf{A}) \cdot n_{R}>3$. Under this assumption, the PEP is minimized if the sum of all the eigenvalues of the product matrix is maximized. For a square matrix, the sum of all the eigenvalues is equal to the trace of the matrix. It can be written as:

$$
\operatorname{tr}(\mathbf{A})=\sum_{k=1}^{n_{T}} \lambda_{k}=\sum_{k=1}^{n_{T}}\left(\sum_{q=0}^{L_{f}-1}\left|e_{t+q}^{k}-s_{t+q}^{k}\right|^{2}\right)
$$

For each pair of coded frames, a matrix $\mathbf{A}$ and then $\operatorname{tr}(\mathbf{A})$ can be computed. The minimum trace is the minimum of all these values $\operatorname{tr}(\mathbf{A})$. Since the product $\operatorname{rank}(\mathbf{A}) \cdot n_{R}>3$, the minimization of the PEP amounts to use a code which has the maximum value of the minimum trace. 


\section{BALANCED CODES}

\section{A. Why balanced codes?}

The concept of "balanced codes" is based on the observation that all the good STTC proposed in the literature present the same property: the generated symbols of the constellation are equally probable.

Indeed, if the binary input data is generated by a memoryless source $\mathbf{S}=\{0,1\}$ with equally probable symbols, then, in the case of 4 - PSK modulation, from a given state $\mathbf{X}=\left[x_{1} x_{2} \ldots x_{L}\right]^{T} \in \mathbb{Z}_{2}^{L}$ of the shift-register realized by $(\nu+1)$ blocks of $n$ bits, the MIMO symbol $\mathbf{Y}=\left[y_{1} y_{2} \ldots y_{n_{T}}\right]^{T} \in \mathbb{Z}_{4}^{n_{T}}$ generated by the STTC encoder shown in Fig.1 is:

$$
\mathbf{Y}=\mathbf{C} \cdot \mathbf{X}
$$

where $\mathbf{C}$ is the generator matrix (1). This is a deterministic relation. Therefore, the STTC is defined by a map:

$$
\Phi: \mathbb{Z}_{2}^{L} \rightarrow \mathbb{Z}_{4}^{n_{T}}
$$

which associates to the state $\mathbf{X}$ an unique codeword $\mathbf{Y}$. Note that $\boldsymbol{\Phi}\left(\mathbb{Z}_{2}^{L}\right) \subseteq \mathbb{Z}_{4}^{n_{T}}$ represents the set of generated codewords $\mathbf{Y}$. A given codeword $\mathbf{Y}$ can be obtained for several states $\mathbf{X} \in \mathbb{Z}_{2}^{L}$. Let $n(\mathbf{Y})$ be the number of occurrences of the codeword $\mathbf{Y}$.

By definition, a STTC is balanced if and only if each generated codeword $\mathbf{Y} \in \boldsymbol{\Phi}\left(\mathbb{Z}_{2}^{L}\right)$ has the same number of occurrences $n(\mathbf{Y})=n_{0} \geq 1$.

In addition, if $\boldsymbol{\Phi}\left(\mathbb{Z}_{2}^{L}\right)=\mathbb{Z}_{4}^{n_{T}}$, then all the codewords are generated and the STTC is fully balanced.

Due to the random source $\mathbf{S}=\{0,1\}$, from a given state $\mathbf{X}$ the encoder can have only 4 equally probable next states. The matrix $\mathbf{T}$ of the transition probabilities between these states corresponds to a Markov chain. Due to the symmetry of the matrix $\mathbf{T}$, the steady state probabilities of the states $\mathbf{X}$ are all equal. For a balanced code, by using (8), the generated codewords $\mathrm{Y}$ are also equally probable. In other words, the generated symbols of the constellation are equally probable.

\section{B. Properties of balanced STTC}

The design of the B-STTC is based on the following properties:

Theorem 1: If a MIMO code with a $L$-length shift-register is fully balanced then $L \geq L_{\min }=n . n_{T}$. One can observe that $L_{\text {min }}=\operatorname{dim}\left(\mathbb{Z}_{2^{n}}^{n_{T}}\right)$.

Theorem 2: Let us consider a balanced MIMO code with a $L$-length shift-register. Then, for any additional column matrix $\mathbf{C}_{i} \in \mathbb{Z}_{2^{n}}^{n_{T}}$, the resulting MIMO code with a $(L+1)$-length shift-register is also balanced.

Definition 1: The vectors $\mathbf{C}_{1}, \mathbf{C}_{2}, \ldots, \mathbf{C}_{L}$ are linearly independent if the equation

$$
x_{1} \mathbf{C}_{1}+x_{2} \mathbf{C}_{2}+\cdots+x_{L} \mathbf{C}_{L}=\mathbf{0} \in \mathbb{Z}_{2^{n}}^{n_{T}}
$$

with $x_{i} \in\{0,1\}$ holds if and only if $x_{i}=0$ for every $i=1,2, \ldots, L$.
Definition 2: A set of linearly independent vectors $\mathbf{C}_{1}, \mathbf{C}_{2}, \ldots, \mathbf{C}_{m}$ is called a base for $\mathbb{Z}_{2^{n}}^{n_{T}}$ if and only if

$\operatorname{span}\left(\mathbf{C}_{1}, \mathbf{C}_{2}, \ldots, \mathbf{C}_{m}\right)=\left\{\sum_{i=1}^{m} x_{i} \mathbf{C}_{i} / x_{i} \in\{0,1\}, i=1,2, \ldots, L\right\}=\mathbb{Z}_{2^{n}}^{n_{T}}$.

In this case, $m=n . n_{T}$ is the dimension of the set $\mathbb{Z}_{2^{n}}^{n_{T}}$.

\section{DESIGN OF 4-PSK STTC WITH 2 TRANSMIT ANTENNAS}

The design of the fully balanced codes includes 2 steps:

- First step: generation of all the bases of $\mathbb{Z}_{4}^{2}$.

- Second step: permutation of the column vectors of each obtained base to generate all the fully balanced codes.

\section{A. Properties of the bases of $\mathbb{Z}_{4}^{2}$}

In the following, the properties of the bases of $\mathbb{Z}_{4}^{2}$, which characterize the fully balanced codes are listed. Further, by using these properties, we can design all the base of $\mathbb{Z}_{4}^{2}$, then the fully balanced codes.

Property 1: One base of $\mathbb{Z}_{4}^{2}$ contains $\operatorname{dim}\left(\mathbb{Z}_{4}^{2}\right)=4$ vectors.

Property 2: The null vector $0 \in \mathbb{Z}_{4}^{2}$ can not be used to form a base.

Property 3: If the vector $\mathbf{C}_{i} \in \mathbb{Z}_{4}^{2}$ is used to form a base, then $\mathbf{C}_{j}=-\mathbf{C}_{i}$ does not belong to this base.

Property 4: If the vectors $\mathbf{C}_{1}, \mathbf{C}_{2}, \ldots, \mathbf{C}_{m} \in \mathbb{Z}_{4}^{2}$ with $m<4$ are linearly independant then the vectors

$$
\mathbf{C}_{m+1}=\sum_{i=1}^{m} \lambda_{i} \mathbf{C}_{i} \in \mathbb{Z}_{4}^{2}, \quad \lambda_{i} \in\{-1,0,1\}
$$

can not be used to obtain $m+1$ linearly independent vectors.

Property 5: $\mathcal{C}_{0}=2 \mathbb{Z}_{2}^{2}$ is a normal subgroup of the additive group $\mathbb{Z}_{4}^{2}$. For each element $v \in \mathbb{Z}_{2}^{2}$ we consider the coset $\mathcal{C}_{v}=v+2 \mathbb{Z}_{2}^{2}$, where addition is in $\mathbb{Z}_{4}^{2}$. Then, $\mathbb{Z}_{4}^{2}$ is divided into 4 cosets.

Property 6: If $u_{1} \in \mathcal{C}_{u}$ and $v_{1} \in \mathcal{C}_{v}$ then $u_{1}+v_{1} \in \mathcal{C}_{u \oplus v}$ where $\oplus$ represents the addition in $\mathbb{Z}_{2}^{2}$.

Property 7: If $u_{1} \in \mathcal{C}_{u}$ then $u_{1}+\mathcal{C}_{v}=\mathcal{C}_{u \oplus v}$.

Property 8: $u+\mathcal{C}_{u}=\mathcal{C}_{0}=2 \mathbb{Z}_{2}^{2}$.

Property 9: The sum of two cosets is defined by

$$
\mathcal{C}_{u}+\mathcal{C}_{v}=\left\{u_{1}+v_{1} / u_{1} \in \mathcal{C}_{u} \text { and } v_{1} \in \mathcal{C}_{v}\right\}
$$

Property 10: The direct sum of two cosets is a coset:

$$
\mathcal{C}_{u}+\mathcal{C}_{v}=\mathcal{C}_{u \oplus v}
$$

Property 11: If $u_{1} \in \mathcal{C}_{u}$ then $-u_{1} \in \mathcal{C}_{u}$.

Property 12: If $p_{1}, p_{2} \in \mathbb{Z}_{2}^{2} \backslash\{0\}$ and $p_{1} \neq p_{2}$, then $\operatorname{span}\left(2 p_{1}, 2 p_{2}\right)=2 \mathbb{Z}_{2}^{2}=\mathcal{C}_{0}$.

Property 13: If $p_{i} \in \mathbb{Z}_{2}^{2}$ then the sum of 2 different elements of the coset $\mathcal{C}_{p_{i}}$ is an element of $\mathcal{C}_{0} \backslash\left\{2 p_{i}\right\}$, for $i=1,2,3$.

Property 14: One base of $\mathbb{Z}_{4}^{2}$ contains at least one vectors in $\mathcal{C}_{0}$.

Property 15: One base of $\mathbb{Z}_{4}^{2}$ contains at most 2 vectors in the same coset.

Property 16: One base of $\mathbb{Z}_{4}^{2}$ always contains 2 vectors which belong to $2 \operatorname{cosets} \mathcal{C}_{p_{1}}, \mathcal{C}_{p_{2}}$ different from $\mathcal{C}_{0}$ such as the vectors $p_{1}, p_{2}$ are linearly independent. Similarly, the cosets $\mathcal{C}_{p_{1}}, \mathcal{C}_{p_{2}}$ are called linearly independent. 
One base of $\mathbb{Z}_{4}^{2}$ contains at least one vector in $\mathcal{C}_{0}$ and at most 2 vectors in $\mathcal{C}_{0}$ (Property 14, 15). Then, there are two types of fully balanced codes of $\mathbb{Z}_{4}^{2}$ :

- Type $I$ codes which contain only 1 non-null vector in $\mathcal{C}_{0}$

- Type $I I$ codes which contain 2 non-null vectors in $\mathcal{C}_{0}$

\section{B. Design of fully balanced codes of type $I$}

A base of a fully balanced code of type I contains only one vector $2 p_{i} \in \mathcal{C}_{0} \backslash\{0\}=\mathcal{C}_{0}^{*}$. A second vector $2 p_{j} \in \mathcal{C}_{0}^{*}$ is obtained as the sum of 2 different vectors of a $\operatorname{coset} \mathcal{C}_{p_{j}}$ : there are 3 possibilities to choose this coset. Due to the structure of a coset $\mathcal{C}_{p_{j}}=\left\{p_{j},-p_{j}, q_{j},-q_{j}\right\}$, there are 4 different ways to choose these 2 vectors : $\left\{p_{j}, q_{j}\right\},\left\{-p_{j}, q_{j}\right\},\left\{p_{j},-q_{j}\right\}$, $\left\{-p_{j},-q_{j}\right\}$. The last vector can be chosen in a different coset, so there are $2 \cdot 4=8$ possibilities. Therefore, there are $3 \cdot 4 \cdot 8=96$ different bases of type $\mathrm{I}$ in $\mathbb{Z}_{4}^{2}$.

\section{Design of fully balanced codes of type II}

A base of a fully balanced coset code of type $I I$ contains 2 different vectors in $\mathcal{C}_{0}^{*}=2 \mathbb{Z}_{2}^{2} \backslash\{0\}$. These 2 vectors generate $\mathcal{C}_{0}=2 \mathbb{Z}_{2}^{2}$. Therefore, it is sufficient to choose a vector $u_{1}$ in a coset $\mathcal{C}_{u}$ to obtain the whole $\operatorname{coset} \mathcal{C}_{u}$. In the same way, if we choose another vector $v_{1}$ in a $\operatorname{coset} \mathcal{C}_{v}$, the whole coset $\mathcal{C}_{v}$ is also generated. Moreover, $u_{1}+v_{1} \in \mathcal{C}_{u \oplus v}$. Therefore, the sum of the vector $u_{1}+v_{1}$ with the coset $\mathcal{C}_{0}$ gives $\mathcal{C}_{u \oplus v}$. Hence, all the vectors of $\mathbb{Z}_{4}^{2}$ are generated.

The algorithm to obtain a base of type II is as follows:

1) Choose 2 different vectors in $\mathcal{C}_{0}^{*}$ (3 possibilities);

2) Choose 2 different cosets $\mathcal{C}_{u}$ and $\mathcal{C}_{v}$ different from $\mathcal{C}_{0}$ (3 possibilities);

3) For each of these 2 cosets, choose one representative (4 possibilities for each coset).

Therefore, there are $3 \cdot 3 \cdot 4 \cdot 4=144$ bases of type $I I$ in $\mathbb{Z}_{4}^{2}$. Finally, the total number of the bases of $\mathbb{Z}_{4}^{2}$ is: $96+144=240$ bases.

\section{CODE PERFORMANCE}

Before showing all the best codes based on the trace criterion, we propose herein some trace properties of 4-state 4-PSK STTC:

- Property $P_{1}$ : the codes $\mathbf{C}=\left[\begin{array}{lll}\mathbf{C}_{1} \mathbf{C}_{2} & \mathbf{C}_{3} \mathbf{C}_{4}\end{array}\right]$, $\mathbf{C}^{\prime}=\left[-\mathbf{C}_{1} \mathbf{C}_{2} \quad \mathbf{C}_{3} \mathbf{C}_{4}\right]$ and $\mathbf{C}^{\prime \prime}=\left[\begin{array}{ll}\left.\mathbf{C}_{1} \mathbf{C}_{2}-\mathbf{C}_{3} \mathbf{C}_{4}\right] \text { have } & \end{array}\right.$ the same minimum trace value.

- Property $P_{2}$ : the codes $\mathbf{C}=\left[\begin{array}{lll}\mathbf{C}_{1} \mathbf{C}_{2} & \mathbf{C}_{3} \mathbf{C}_{4}\end{array}\right]$ and $\mathbf{C}^{\prime}=\left[\begin{array}{l|l}\mathbf{C}_{2} \mathbf{C}_{1} & \mathbf{C}_{3} \mathbf{C}_{4}\end{array}\right]$ have the same minimum trace value.

- Property $P_{3}:$ the codes $\mathbf{C}=\left[\begin{array}{lll}\mathbf{C}_{1} \mathbf{C}_{2} & \mathbf{C}_{3} \mathbf{C}_{4}\end{array}\right]$ and $\mathbf{C}^{\prime}=\left[\begin{array}{lll}\mathbf{C}_{3} \mathbf{C}_{4} & \mathbf{C}_{1} \mathbf{C}_{2}\end{array}\right]$ achieve the same minimum trace value.

- Property $P_{4}$ : the same minimum trace value is obtained by using a permutation between the rows of the generator matrix $\mathbf{C}$, i.e, a permutation between the indices of the transmit antennas.
TABLE I

STRUCTURE OF 4-STATE 4-PSK FULLY BALANCED STTC WITH 2 TRANSMIT ANTENNAS AND $\min (\operatorname{TR}(\mathbf{A}))=10$

$$
\mathbf{C}=\left[\begin{array}{llll}
2 & 0 & 2 & 1 \\
1 & 2 & 0 & 2
\end{array}\right] \quad \mathbf{C}^{\prime}=\left[\begin{array}{llll}
0 & 2 & 2 \\
2 & 1 & 2 & \left.\mathbf{C}_{4}\right] \forall \mathbf{C}_{4} \in\left\{\left[\begin{array}{l}
1 \\
1
\end{array}\right],\left[\begin{array}{l}
1 \\
3
\end{array}\right]\right\}
\end{array}\right.
$$

An exhaustive computer search is carried out to detect all the 4-state 4-PSK STTC with 2 transmit antennas that achieve the maximum rank and the maximum trace. A set of 80 codes with $\min (\operatorname{rank}(\mathbf{A}))=2$ and $\min (\operatorname{tr}(\mathbf{A}))=10$ is found. All these codes offer a minimum product distance $d_{p}^{2}=4 \cdot 6=24$ which is the best product distance that can be achieved for 4-state 4-PSK STTC with 2 transmit antennas. Besides, it can be observed that all these codes are fully balanced STTC of type II. Since the minimum trace value has been obtained, all these 80 codes can be generated by using Table I with the trace properties $P_{1}$ to $P_{4}$. As an example, if we choose from Table I the generator matrix $\mathbf{C}$, by using the properties $P_{1}$ to $P_{3}$, we obtain the generator matrix $\left[\begin{array}{llll}0 & 2 & 1 & 2 \\ 2 & 3 & 2 & 0\end{array}\right]$ (in bold in Tab. II) proposed by Chen [2].

The distance spectrum of all these 80 codes has been also computed for $L_{f}=3$. Among them, there are 16 codes which achieve 10 as the minimum euclidian distance with the multiplicity 4 whereas the other 64 vectors have 10 as the minimum euclidian distance with the multiplicity 6 . Then, all these 16 4-state fully balanced codes which offer the best performance over fast and slow Rayleigh fading channels with two or more receive antennas is given in Tab. II below.

TABLE II

4-STATE 4-PSK FULLY BALANCED STTC WITH 2 TRANSMIT ANTENNAS $\operatorname{AND} \min (\operatorname{TR}(\mathbf{A}))=10$

\begin{tabular}{|c|c|c|c|c|c|c|c|c|c|c|c|c|c|c|c|}
\hline$\left[\begin{array}{l}1 \\
2\end{array}\right.$ & $\begin{array}{l}2 \\
0\end{array}$ & $\begin{array}{l}0 \\
2\end{array}$ & $\left.\begin{array}{l}2 \\
1\end{array}\right]$ & {$\left[\begin{array}{l}1 \\
2\end{array}\right.$} & $\begin{array}{l}2 \\
0\end{array}$ & $\begin{array}{l}0 \\
2\end{array}$ & $\left.\begin{array}{l}2 \\
3\end{array}\right]$ & {$\left[\begin{array}{l}3 \\
2\end{array}\right.$} & $\begin{array}{l}2 \\
0\end{array}$ & $\begin{array}{l}0 \\
2\end{array}$ & $\left.\begin{array}{l}2 \\
1\end{array}\right]$ & {$\left[\begin{array}{l}3 \\
2\end{array}\right.$} & $\begin{array}{l}2 \\
0\end{array}$ & $\begin{array}{l}0 \\
2\end{array}$ & $\left.\begin{array}{l}2 \\
3\end{array}\right]$ \\
\hline$\left[\begin{array}{l}2 \\
0\end{array}\right.$ & $\begin{array}{l}1 \\
2\end{array}$ & $\begin{array}{l}2 \\
1\end{array}$ & $\left.\begin{array}{l}0 \\
2\end{array}\right]$ & {$\left[\begin{array}{l}2 \\
0\end{array}\right.$} & $\begin{array}{l}1 \\
2\end{array}$ & $\begin{array}{l}2 \\
3\end{array}$ & $\left.\begin{array}{l}0 \\
2\end{array}\right]$ & {$\left[\begin{array}{l}2 \\
0\end{array}\right.$} & $\begin{array}{l}3 \\
2\end{array}$ & $\begin{array}{l}2 \\
1\end{array}$ & $\left.\begin{array}{l}0 \\
2\end{array}\right]$ & {$\left[\begin{array}{l}2 \\
0\end{array}\right.$} & $\begin{array}{l}3 \\
2\end{array}$ & $\begin{array}{l}2 \\
3\end{array}$ & $\left.\begin{array}{l}0 \\
2\end{array}\right]$ \\
\hline$\left[\begin{array}{l}2 \\
1\end{array}\right.$ & $\begin{array}{l}0 \\
2\end{array}$ & $\begin{array}{l}2 \\
0\end{array}$ & $\left.\begin{array}{l}1 \\
2\end{array}\right]$ & {$\left[\begin{array}{l}2 \\
1\end{array}\right.$} & $\begin{array}{l}0 \\
2\end{array}$ & $\begin{array}{l}2 \\
0\end{array}$ & $\left.\begin{array}{l}3 \\
2\end{array}\right]$ & {$\left[\begin{array}{l}2 \\
3\end{array}\right.$} & $\begin{array}{l}0 \\
2\end{array}$ & $\begin{array}{l}2 \\
0\end{array}$ & $\left.\begin{array}{l}1 \\
2\end{array}\right]$ & {$\left[\begin{array}{l}2 \\
3\end{array}\right.$} & $\begin{array}{l}0 \\
2\end{array}$ & $\begin{array}{l}2 \\
0\end{array}$ & $\left.\begin{array}{l}3 \\
2\end{array}\right]$ \\
\hline$\left[\begin{array}{l}0 \\
2\end{array}\right.$ & $\begin{array}{l}2 \\
1\end{array}$ & $\begin{array}{l}1 \\
2\end{array}$ & $\left.\begin{array}{l}2 \\
0\end{array}\right]$ & {$\left[\begin{array}{l}0 \\
2\end{array}\right.$} & $\begin{array}{l}2 \\
1\end{array}$ & $\begin{array}{l}3 \\
2\end{array}$ & $\left.\begin{array}{l}2 \\
0\end{array}\right]$ & {$\left[\begin{array}{l}0 \\
2\end{array}\right.$} & $\begin{array}{l}2 \\
3\end{array}$ & $\frac{1}{2}$ & $\left.\begin{array}{l}\mathbf{2} \\
\mathbf{0}\end{array}\right]$ & {$\left[\begin{array}{l}0 \\
2\end{array}\right.$} & $\begin{array}{l}2 \\
3\end{array}$ & $\begin{array}{l}3 \\
2\end{array}$ & $\left.\begin{array}{l}2 \\
0\end{array}\right]$ \\
\hline
\end{tabular}

In this table, all the codes are related due to the trace properties presented before. The codes of the third line are obtained from the codes of the first line by permuting the indices of the antennas, i.e. the lines of the generator matrix C. In the same way, the codes of the fourth line are obtained from the codes of the second line of the table.

In order to confirm the utility of the fully balanced STTC, an exhaustive computer search of all 4-state STTC has also been carried out. The obtained results confirm that Table II contains all the best STTC. There are not other codes with better performance than the codes given in this table.

For the 16-state 4-PSK STTC, the construction and the research of the best codes are made from the 4-state 4-PSK STTC by using Theorem 2. Table III herein contains all the 
TABLE III

16-STATE 4-PSK FULLY BALANCED STTC WITH 2 TRANSMIT ANTENNAS $\operatorname{AND} \min (\operatorname{TR}(\mathbf{A}))=16$

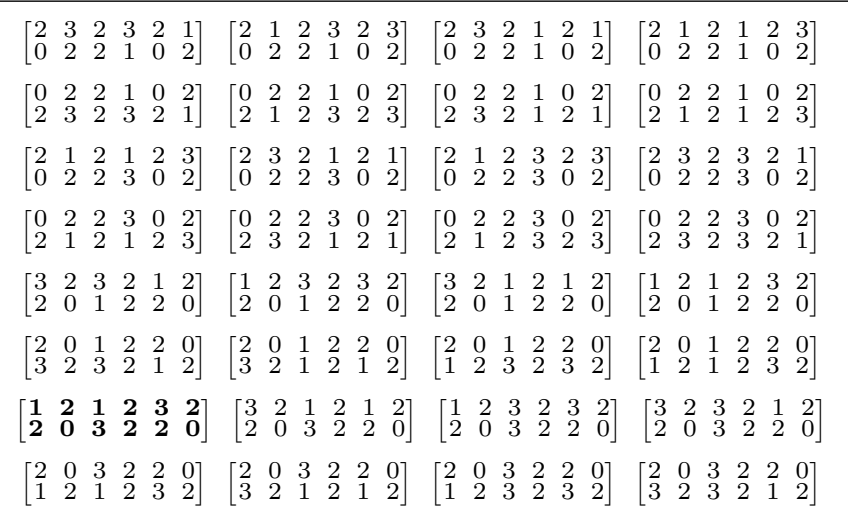

16-state Balanced STTC which offer the best performance over fast and slow Rayleigh fading channels with two or more receive antennas. All these codes have $\min (\operatorname{tr}(\mathbf{A}))=16$ and offer a minimum product distance $d_{p}^{2}=128$. Among them, we found the code proposed by Chen $\left[\begin{array}{llllll}1 & 2 & 1 & 2 & 3 & 2 \\ 2 & 0 & 3 & 2 & 2 & 0\end{array}\right]$ (in bold in Tab. III).

Finally, the performance of all these 4-state and 16-state codes for 2 transmit antennas is evaluated by simulation with 1 and 2 receive antennas over fast Rayleigh fading channels. Each simulated frame consists of 128 symbols transmitted from each transmit antennas. The Frame Error Rate (FER) performance are shown in Fig. 2.

Note that all the codes given in Tab. II achieve the same performance, i.e. the performance of the Chen's code [2] : full rank, $\min (\operatorname{tr}(\mathbf{A}))=10, d_{p}^{2}=24$. These codes outperform the codes proposed in [7]

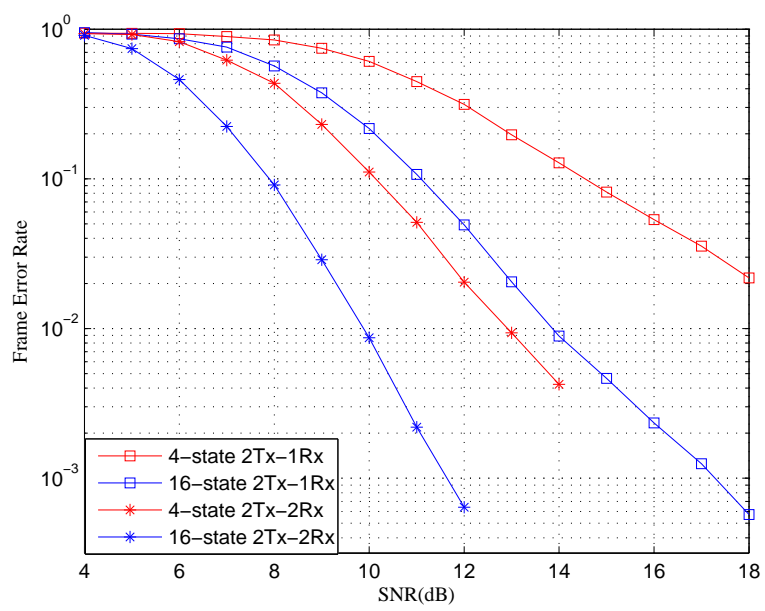

Fig. 2. FER performance of the 4-PSK STTC with two transmit antennas

\section{CONCLUSION}

In this paper, a new class of 4-PSK STTC for two transmit antennas has been proposed. These codes generate the points of the constellation with the same probability. It has been shown that the best STTC belong to this class. Therefore, the systematic search for good codes can be drastically reduced to this class. The design of these balanced codes has been described. A complete list of the best 4-state codes and several 16-state codes for 2 transmit antennas have also been given. All the fully balanced STTC listed in this paper are equivalent, i.e. they have the same rank, trace, product distance and distance spectrum. The simulation results have shown that they outperform the other STTC for 2 transmit antennas.

\section{REFERENCES}

[1] V. Tarokh, N. Seshadri, and A. Calderbank, "Space-time codes for high data rate wireless communication: Performance criterion and code construction," IEEE Trans. Inform. Theory, vol. 44, no. 2, pp. 744-765, March 1998.

[2] Z. Chen, J. Yuan, and B. Vucetic, "Improved space-time trellis coded modulation scheme on slow fading channels," Electron. Lett., vol. 37, no. 7, pp. 440-441, March 2001.

[3] T. Liew and L. Hanzo, "Space-time codes and concatenated channel codes for wireless communications," Proceedings of the IEEE, vol. 90, no. 2, pp. 187-219, Feb 2002.

[4] W. Firmanto, B. Vucetic, and J. Yuan, "Space-time TCM with improved performance on fast fading channels," IEEE Commun. Lett., vol. 5, no. 4, pp. 154-156, April 2001.

[5] S. Baro, G. Bauch, and A. Hansmann, "Improved codes for space-time trellis-coded modulation," IEEE Commun. Lett., vol. 4, no. 1, pp. 20-22, Jan. 2000.

[6] D. Ionescu, K. Mukkavilli, Y. Zhiyuan, and J. Lilleberg, "Improved 8- and 16-state space-time codes for 4PSK with two transmit antennas," IEEE Commun. Lett., vol. 5, no. 7, pp. 301-303, July 2001.

[7] Y. Hong and A. G. i Fabregas, "New Space-Time Trellis Codes for Slow Fading Channels," in Proc. IEEE VTC 2006-Spring, vol. 3, May 2006, pp. 1492 - 1496.

[8] B. Rassool, F. Heliot, L. Revelly, M. Dohler, R. Nakhai, and H. Aghvami, "Fast search techniques for obtaining space-time trellis codes for Rayleigh fading channels and its performance in CDMA systems," in Proc. IEEE VTC 2003-Spring, vol. 1, April 2003, pp. 66-69.

[9] Y. Jung and J. Lee, "Improved space-time trellis codes using expanded signal set in slow Rayleigh fading channels," in Proc. IEEE ISIT'2002, June 2002, p. 248. 